\title{
Development of Mathematical Formulas Correlating the Normal and Tangential Components of Acceleration with Its Rectangular (x-y) Components
}

\author{
Rogelio Fretten C. Dela Cruz ${ }^{*}$ \\ ${ }^{1}$ Malayan Colleges Laguna, PuloDiezmo Road, Cabuyao City, Laguna, Philippines. \\ * Corresponding author. Tel.: 049-832-4000; fax: 049-520-8975; email: rfcdelacruz@mcl.edu.ph \\ Manuscript submitted June 22, 2014; accepted December 15, 2014. \\ doi: 10.17706/ijapm.2015.5.1.39-47
}

\begin{abstract}
In this study, a topic in Engineering Mechanics was examined. The mathematical formulas correlating the normal and tangential components, $a_{n}$ and $a_{t}$, of acceleration with its rectangular components, $a_{x}$ and $a_{y}$, were derived. This study will provide engineering practitioners, teachers, and students with additional tools in solving problems in curvilinear motions.
\end{abstract}

Key words: Curvilinear motion, normal and tangential components of acceleration, rectangular components.

\section{Introduction}

In curvilinear motions, it is very useful to resolve the acceleration into components, either into its normal and tangential components, which are normal and tangent to the path of the motion, or into its rectangular components, commonly known as $x$ and $y$ components. These components separate and denote respectively the rate of change of magnitude and of direction of velocity. They are particularly useful when it is needed to relate velocity and acceleration directly with the path itself [1].

In the context of this study, acceleration is the rate at which the velocity of a body changes per unit of time. Velocity is the rate of linear motion of a body in a particular direction. The magnitude of velocity, known as speed, is usually expressed in terms of distance covered per unit of time. The mathematical definition of

velocity is, $v=\frac{d s}{d t}$, and for acceleration, $a=\frac{d v}{d t}$, where $v$ is velocity, $a$ is acceleration, $s$ is position, and $t$ is time [1]-[5].

The main objective of this study is to derive the mathematical formulas correlating the normal and tangential components, $a_{n}$ and $a_{t}$, of acceleration with its rectangular components, $a_{x}$ and $a_{y}$. The specific objectives are:

1) To develop mathematical formulas correlating the normal and tangential components of acceleration with its rectangular components when the angle $\theta$ is measured between $a_{x}$ and $a_{t}$.

2) To develop mathematical formulas correlating the normal and tangential components of acceleration with its rectangular components when the angle $\theta$ is measured between $a_{x}$ and $a_{n}$.

For the scope and limitation, the study covered the derivation of mathematical formulas correlating the normal and tangential components of acceleration with its rectangular components. All discussions referred to curvilinear motion in a plane, but may also be applicable to spatial motion.

The significance of this study is to provide alternative solutions to problems in curvilinear motions. These 
formulas will serve as additional tools for engineering practitioners, teachers, and students.

\section{Curvilinear Motion}

In curvilinear motion, the position of the particle is specified by the coordinate $s$, which is the distance measured along the path from a fixed reference point. As the particle moves from $A$ to $B$, see Fig. 1, during an infinitesimal time interval $d t$, it traces an arc of radius $\rho$ and infinitesimal length $d s[2]-[8]$. where, $d s=\rho d \theta$

hence, $s=\rho \theta$

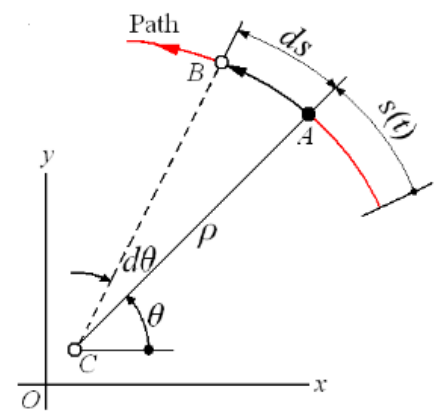

Fig. 1. Motion path of particle.

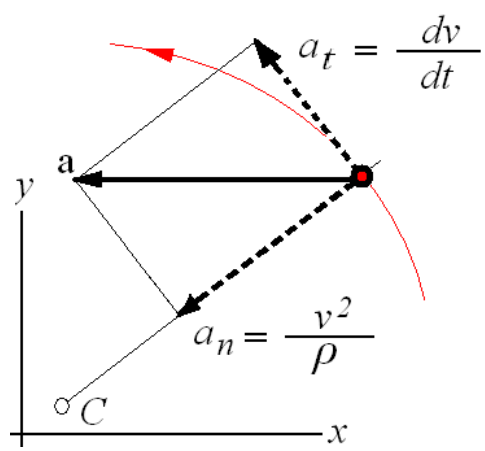

Fig. 2. Normal and tangential components of acceleration.

where $\theta$ is in radians, and $\rho$ is called the radius of curvature of the path at $A$.

If the equation of the path is known, its radius of curvature can be computed from:

$$
\rho=\frac{\left[1+\left(\frac{d y}{d x}\right)^{2}\right]^{3 / 2}}{\left|\frac{d^{2} y}{d x^{2}}\right|} \text { or } \rho=\frac{\left[1+\left(\frac{d x}{d y}\right)^{2}\right]^{3 / 2}}{\left|\frac{d^{2} x}{d y^{2}}\right|} \text {. }
$$

The normal and tangential components of acceleration, see in Fig. 2, are expressed as follows,

Normal component of acceleration $a, a_{n}=\frac{v^{2}}{\rho}$.

Tangential component of acceleration a, $a_{t}=\frac{d v}{d t}$.

Also, $a_{t}=v \frac{d v}{d s}$ 
It is very useful to resolve the acceleration into components, either into its normal and tangential components, which are normal and tangent to the path of the motion, or into its rectangular components, commonly known as $x$ and $y$ components. In this regard, it is important to establish the correlation between these components.

\subsection{When the Angle $\theta$ Is Measured between $a_{x}$ and $a_{t}$}

Derivation of mathematical formulas correlating the normal and tangential components of acceleration with its rectangular components when the angle $\theta$ is measured between $a_{x}$ and $a_{t}$, see in Fig. 3 . where:

$a_{t}$ is the tangential component of acceleration

$a_{n}$ is the normal component of acceleration

$a_{x}$ is the $x$-component of acceleration

$a_{y}$ is the $y$-component of acceleration

$a$ is the resultant acceleration

See Fig. 4, we get

$$
\begin{aligned}
& a_{t}=d_{1}+d_{2} . \\
& a_{y}=c_{1}+c_{2} . \\
& d_{2}=\frac{a_{x}}{\cos \theta} .
\end{aligned}
$$



(a)

(b)

Fig. 3. Motion path of particle.
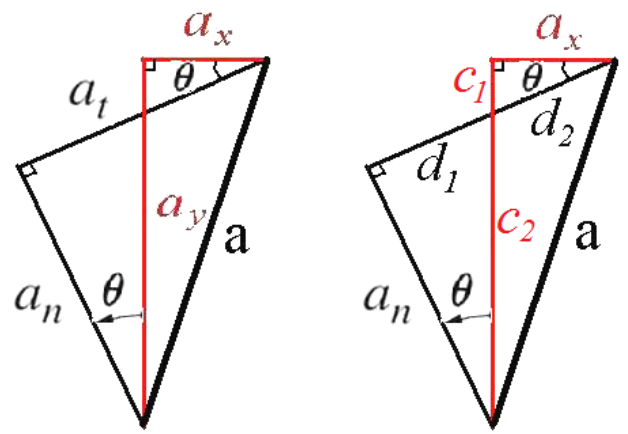

Fig. 4. Acceleration components polygon.

$$
c_{1}=a_{x} \tan \theta \text {. }
$$




$$
\begin{aligned}
& c_{2}=a_{y}-c_{1} \text {. } \\
& c_{2}=a_{y}-a_{x} \tan \theta . \\
& d_{1}=c_{2} \sin \theta \text {. } \\
& d_{1}=\left(a_{y}-a_{x} \tan \theta\right) \sin \theta \text {. } \\
& a_{t}=a_{y} \sin \theta-a_{x} \sin \theta \tan \theta+\frac{a_{x}}{\cos \theta} . \\
& a_{t}=a_{y} \sin \theta-a_{x}\left(\sin \theta \tan \theta-\frac{1}{\cos \theta}\right) \text {. } \\
& a_{t}=a_{y} \sin \theta-a_{x}\left(\frac{\sin ^{2} \theta}{\cos \theta}-\frac{1}{\cos \theta}\right) \text {. } \\
& a_{t}=a_{y} \sin \theta-a_{x}\left(\frac{-\cos ^{2} \theta}{\cos \theta}\right) . \\
& a_{t}=a_{y} \sin \theta+a_{x} \cos \theta \text {. } \\
& \mathrm{a}^{2}=a_{t}^{2}+a_{n}^{2} \text {. } \\
& \mathrm{a}^{2}=a_{x}^{2}+a_{y}^{2} \text {. } \\
& a_{n}{ }^{2}=\mathrm{a}^{2}-a_{t}{ }^{2} . \\
& a_{n}^{2}=a_{x}^{2}+a_{y}{ }^{2}-\left(a_{y} \sin \theta+a_{x} \cos \theta\right)^{2} \text {. } \\
& a_{n}{ }^{2}=a_{y}{ }^{2}-a_{y}{ }^{2} \sin ^{2} \theta-2 a_{x} a_{y} \sin \theta \cos \theta+a_{x}{ }^{2}-a_{x}{ }^{2} \cos ^{2} \theta \text {. } \\
& a_{n}{ }^{2}=a_{y}{ }^{2}\left(1-\sin ^{2} \theta\right)-2 a_{x} a_{y} \sin \theta \cos \theta+a_{x}{ }^{2}\left(1-\cos ^{2} \theta\right) . \\
& a_{n}^{2}=a_{y}{ }^{2} \cos ^{2} \theta-2 a_{x} a_{y} \sin \theta \cos \theta+a_{x}{ }^{2} \sin ^{2} \theta \text {. } \\
& a_{n}^{2}=\left(a_{y} \cos \theta-a_{x} \sin \theta\right)^{2} . \\
& a_{n}=a_{y} \cos \theta-a_{x} \sin \theta \text {. } \\
& c_{2}=\frac{a_{n}}{\cos \theta} \\
& d_{1}=a_{n} \tan \theta \\
& d_{2}=a_{t}-a_{n} \tan \theta \\
& c_{1}=d_{2} \sin \theta \\
& c_{1}=\left(a_{t}-a_{n} \tan \theta\right) \sin \theta . \\
& c_{1}=a_{t} \sin \theta-a_{n} \sin \theta \tan \theta \text {. }
\end{aligned}
$$




$$
\begin{aligned}
& a_{y}=a_{t} \sin \theta-a_{n} \sin \theta \tan \theta+\frac{a_{n}}{\cos \theta} \\
& a_{y}=a_{t} \sin \theta-a_{n}\left(\sin \theta \tan \theta-\frac{1}{\cos \theta}\right) \text {. } \\
& a_{y}=a_{t} \sin \theta-a_{n}\left(\frac{\sin \theta \sin \theta}{\cos \theta}-\frac{1}{\cos \theta}\right) \text {. } \\
& a_{y}=a_{t} \sin \theta-a_{n}\left(\frac{\sin ^{2} \theta-1}{\cos \theta}\right) \text {. } \\
& a_{y}=a_{t} \sin \theta-a_{n}\left(\frac{-\cos ^{2} \theta}{\cos \theta}\right) . \\
& a_{y}=a_{t} \sin \theta+a_{n} \cos \theta \text {. } \\
& a_{x}^{2}=\mathrm{a}^{2}-a_{y}^{2} \\
& a_{x}^{2}=a_{t}^{2}+a_{n}^{2}-\left(a_{t} \sin \theta+a_{n} \cos \theta\right)^{2} . \\
& a_{x}{ }^{2}=a_{t}{ }^{2}-a_{t}{ }^{2} \sin ^{2} \theta-2 a_{t} a_{n} \sin \theta \cos \theta+a_{n}{ }^{2}-a_{n}{ }^{2} \cos ^{2} \theta \text {. } \\
& a_{x}{ }^{2}=a_{t}{ }^{2}\left(1-\sin ^{2} \theta\right)-2 a_{t} a_{n} \sin \theta \cos \theta+a_{n}{ }^{2}\left(1-\cos ^{2} \theta\right) \text {. } \\
& a_{x}^{2}=a_{t}^{2} \cos ^{2} \theta-2 a_{t} a_{n} \sin \theta \cos \theta+a_{n}^{2} \sin ^{2} \theta \text {. } \\
& a_{x}^{2}=\left(a_{t} \cos \theta-a_{n} \sin \theta\right)^{2} . \\
& a_{x}=a_{t} \cos \theta-a_{n} \sin \theta
\end{aligned}
$$

\subsection{When the Angle $\theta$ Is Measured between $a_{x}$ and $a_{n}$}

Derivation of mathematical formulas correlating the normal and tangential components of acceleration with its rectangular components when the angle $\theta$ is measured between $a_{x}$ and $a_{n}$, see in Fig. 5.



Fig. 5. Motion path of particle.

See Fig. 6, we get

$$
a_{t}=c_{1}+c_{2} \text {. }
$$




$$
\begin{aligned}
& a_{x}=d_{1}+d_{2} \text {. } \\
& c_{1}=\frac{a_{y}}{\cos \theta} . \\
& d_{1}=c_{1} \sin \theta \text {. } \\
& d_{1}=\frac{a_{y}}{\cos \theta} \sin \theta \\
& d_{1}=a_{y} \tan \theta \text {. } \\
& d_{2}=a_{x}-a_{y} \tan \theta \text {. } \\
& c_{2}=d_{2} \sin \theta \text {. } \\
& a_{t}=\frac{a_{y}}{\cos \theta}+\left(a_{x}-a_{y} \tan \theta\right) \sin \theta . \\
& a_{t}=a_{x} \sin \theta+\frac{a_{y}}{\cos \theta}-a_{y} \tan \theta \sin \theta \\
& a_{t}=a_{x} \sin \theta+\frac{a_{y}}{\cos \theta}-a_{y} \tan \theta \sin \theta . \\
& a_{t}=a_{x} \sin \theta+a_{y}\left(\frac{1-\sin ^{2} \theta}{\cos \theta}\right) \text {. } \\
& a_{t}=a_{x} \sin \theta+a_{y}\left(\frac{\cos ^{2} \theta}{\cos \theta}\right) . \\
& a_{t}=a_{x} \sin \theta+a_{y} \cos \theta \text {. } \\
& a_{n}^{2}=\mathrm{a}^{2}-a_{t}^{2} . \\
& a_{n}^{2}=a_{x}^{2}+a_{y}^{2}-\left(a_{x} \sin \theta+a_{y} \cos \theta\right)^{2} \text {. } \\
& a_{n}^{2}=a_{x}{ }^{2}-a_{x}{ }^{2} \sin ^{2} \theta-2 a_{x} a_{y} \sin \theta \cos \theta+a_{y}{ }^{2}-a_{y}{ }^{2} \cos ^{2} \theta \text {. } \\
& a_{n}^{2}=a_{x}^{2}\left(1-\sin ^{2} \theta\right)-2 a_{x} a_{y} \sin \theta \cos \theta+a_{y}^{2}\left(1-\cos ^{2} \theta\right) \text {. } \\
& a_{n}^{2}=a_{x}^{2} \cos ^{2} \theta-2 a_{x} a_{y} \sin \theta \cos \theta+a_{y}{ }^{2} \sin ^{2} \theta \text {. } \\
& a_{n}^{2}=\left(a_{x} \cos \theta-a_{y} \sin \theta\right)^{2} . \\
& a_{n}=a_{x} \cos \theta-a_{y} \sin \theta \text {. } \\
& d_{2}=\frac{a_{n}}{\cos \theta} \\
& c_{2}=a_{n} \tan \theta \text {. } \\
& d_{1}=c_{1} \sin \theta \text {. }
\end{aligned}
$$




$$
\begin{gathered}
c_{1}=a_{t}-a_{n} \tan \theta . \\
d_{1}=\left(a_{t}-a_{n} \tan \theta\right) \sin \theta . \\
d_{1}=a_{t} \sin \theta-a_{n} \sin \theta \tan \theta . \\
d_{1}=a_{t} \sin \theta-a_{n} \frac{\sin ^{2} \theta}{\cos \theta} .
\end{gathered}
$$

Substitute (64) and (70) in(44), hence

$$
\begin{gathered}
a_{x}=a_{t} \sin \theta-a_{n} \frac{\sin ^{2} \theta}{\cos \theta}+\frac{a_{n}}{\cos \theta} . \\
a_{x}=a_{t} \sin \theta+a_{n}\left(\frac{1-\sin ^{2} \theta}{\cos \theta}\right) . \\
a_{x}=a_{t} \sin \theta+a_{n} \cos \theta . \\
a_{y}{ }^{2}=a_{t}{ }^{2}+a_{n}{ }^{2}-\left(a_{t} \sin \theta+a_{n} \cos \theta\right)^{2} . \\
a_{y}{ }^{2}=a_{t}{ }^{2}-a_{t}{ }^{2} \sin ^{2} \theta-2 a_{t} a_{n} \sin \theta \cos \theta+a_{n}{ }^{2}-a_{n}{ }^{2} \cos ^{2} \theta . \\
a_{t}{ }^{2}\left(1-\sin ^{2} \theta\right)-2 a_{t} a_{n} \sin \theta \cos \theta+a_{n}{ }^{2}\left(1-\cos ^{2} \theta\right) . \\
a_{t}{ }^{2} \cos ^{2} \theta-2 a_{t} a_{n} \sin \theta \cos \theta+a_{n}{ }^{2} \sin ^{2} \theta . \\
a_{y}{ }^{2}=\left(a_{t} \cos \theta-a_{n} \sin \theta\right)^{2} . \\
a_{y}=a_{t} \cos \theta-a_{n} \sin \theta .
\end{gathered}
$$
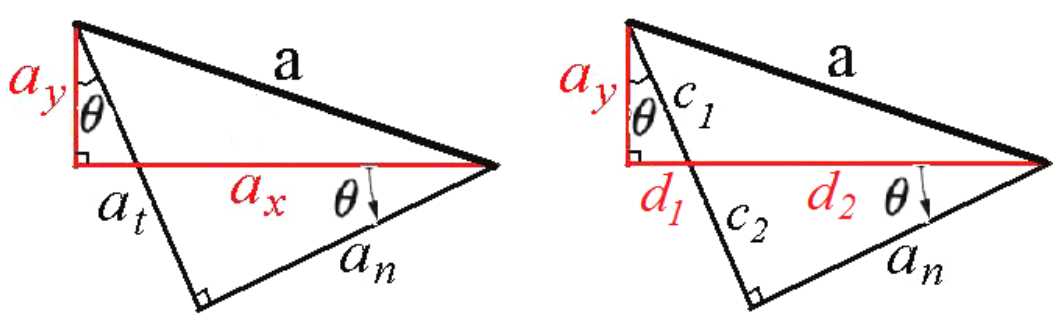

Fig. 6. Acceleration components polygon.

\section{Conclusion and Directions for Future Use}

The mathematical formulas derived correlating the normal and tangential components of acceleration with its rectangularcomponents when the angle $\theta$ is measured between $a_{x}$ and $a_{t}$, see Fig. 3 , are the following:

$$
a_{t}=a_{y} \sin \theta+a_{x} \cos \theta .
$$




$$
\begin{gathered}
a_{n}=a_{y} \cos \theta-a_{x} \sin \theta . \\
a_{x}=a_{t} \cos \theta-a_{n} \sin \theta . \\
a_{y}=a_{t} \sin \theta+a_{n} \cos \theta .
\end{gathered}
$$

And the mathematical formulas correlating the normal and tangential components of acceleration with its rectangularcomponents when the angle $\theta$ is measured between $a_{x}$ and $a_{n}$, see Fig. 5, are as follows:

$$
\begin{aligned}
& a_{t}=a_{x} \sin \theta+a_{y} \cos \theta . \\
& a_{n}=a_{x} \cos \theta-a_{y} \sin \theta . \\
& a_{x}=a_{t} \sin \theta+a_{n} \cos \theta . \\
& a_{y}=a_{t} \cos \theta-a_{n} \sin \theta .
\end{aligned}
$$

The researcher recommends the use of the above mentioned formulas as alternative method in solving problems involving accelerations in curvilinear motions.

\section{Acknowledgment}

This research was inspired by the formulas developed by F. L. Singer in his book [1]. I thank him for that. Also, I would like to thank the following: my wife, Flordeliza R. Dela Cruz and my daughter, MelannieFlor Irene R. Dela Cruz, for the typing job and drawings; and to my endearing dean, Dr. Joy G. Hofileña, for her continuous warm support that inspired me in doing this paper. I would like to express my most sincere gratitude to Malayan Colleges Laguna, Philippines, for funding the publication and presentation of this paper, and above all, I thank God, the Father Almighty, without Him, I am nothing.

This work is dedicated to my mother, Irene C. Dela Cruz, for her continuous tender loving care and to my mother-in-law, Filipinas R. Ruiz for always believing in me.

\section{References}

[1] Singer, F. L. (1980). Engineering Mechanics: Statics and Dynamics (3rd ed.). (pp. 327-369). New York, USA: Harper \& Row, Publishers Inc.

[2] Pytel, A., \& Kiusalaas, J. (2011). Understanding Engineering Mechanics: Dynamics (SI ed.). (pp. 69-82). Singapore: Cengage Learning Asia Pte. Ltd.

[3] Beer, F. P., \& Johnston Jr., E. R. (2007). Vector Mechanics for Engineers: Dynamics (8th ed.). (chapter 11). New York: The McGraw-Hill Co.

[4] Hibbeler, R. C. (2006). Principles of Statics and Dynamics (10th ed.). (pp. 667-684). New York: Prentice-Hall.

[5] Hibbeler, R. C. (2006). Engineering Mechanics: Principles of Statics and Dynamics (pp. 23-27). New Jersey: Pearson Prentice Hall.

[6] Roberts, A. P. (2003). Statics and Dynamics with Background Mathematics. New York: Cambridge University Press.

[7] McGill, D. J., \& King, W. W. (1995). Engineering Mechanics (3rd ed.). Boston: PWS.

[8] Soutas-Little, R. W., Inman, D. J., \& Balint, D. S. (2008). Engineering Mechanics: Dynamics (Computational ed.). (pp. 35-54). USA. 


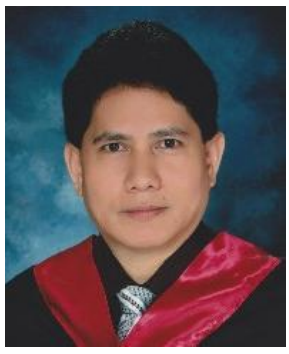

Rogelio Fretten C. Dela Cruz is a graduate of the bachelor of science in civil engineering from Saint Louis University, Baguio City, Philippines (1985) and the master of science in mathematics from Pamantasanng Cabuyao (University of Cabuyao), Cabuyao City, Philippines (2013).

He worked in the Department of Public Works and Highways - La Union Engineering District (1986-1990), then joined San Miguel Packaging Specialists Inc. - Rightpak Plant (1990-2008). He also worked in Mastoura Contracting Company in Ad Dammam, KSA (2008) and later in Vazquez - Madrigal \& Co., Inc. He started in the field of academe as a part-time faculty member in Laguna College of Business and Arts (2005-2010), a full-time faculty in Colegio de San Juan de Letran - Calamba (2009-2012), a part-time faculty in Lyceum of the Philippines - Laguna (2010), part-time faculty in University of Perpetual Help System Laguna (2010-2012), a part-time faculty in Pamantasan ng Cabuyao (2012), and currently, he is an asst. professor and a pollution control officer in Malayan Colleges Laguna, Philippines (2012-present).

Engr. Dela Cruz is a member of Philippine Institute of Civil Engineers (PICE), Mathematical Society of the Philippines - CALABARZON Chapter (MSPC), and Pollution Control Association of the Philippines, Inc, (PCAPI). 